viewed directly in the MMCD interface or downloaded as a tab-delimited file for viewing with spreadsheet software.

We have also built a 'Miscellanea' search engine that allows users to filter results by the biological species, the type of database to be searched or other criteria. These options allow users to rapidly locate particular sets of records or limit their queries to a preferred subset of records.

In summary, MMCD is a practical tool for expediting the time-consuming steps of identifying and researching small molecules. This freely available resource is compatible with both NMR and MS data (singly or in combination) and facilitates high-throughput metabolomics investigations. Ongoing MMCD support is provided by the National Magnetic Resonance Facility at Madison. Users are encouraged to submit data to the BMRB (supported by the National Library of Medicine, Bethesda, MD, USA), which maintains one of the growing data archives that the MMCD relies upon ${ }^{10}$.

Note: Supplementary information is available on the Nature Biotechnology website.

ACKNOWLEDGMENTS

This work was supported by National Institutes of Health grants R21 DK070297 (MRS, PI) and P41
RR02301 (J.L.M.). We are indebted to W.W. Cleland, H. Lardy and L. Anderson for access to their chemical compound collections. We thank Joe Porwall from Sigma-Aldrich for assistance in locating compounds, Zsolt Zolnai for development of the Sesame LIMS and Eldon L. Ulrich for help in coordinating MMC activities with the BMRB.

Qiu Cui, Ian A Lewis, Adrian D Hegeman, Mark E Anderson, Jing Li, Christopher F Schulte, William M Westler, Hamid R Eghbalnia, Michael R Sussman, \& John L Markley

Department of Biochemistry, University of Wisconsin-Madison, 433 Babcock Drive,

Madison, Wisconsin 53706, USA.

e-mail:markley@nmrfam.wisc.edu

1. Mendes, P. Brief Bioinform. 3, 134-145 (2002).

2. Nicholson, J.K., Lindon, J.C. \& Holmes, E. Xenobiotica 29, 1181-1189 (1999).

3. Pauling, L., Robinson, A.B., Teranishi, R. \& Cary, P. Proc. Natl Acad. Sci. USA 68, 2374-2376 (1971).

4. Viant, M.R., Rosenblum, E.S. \& Tieerdema, R.S. Environ. Sci Technol. 37, 4982-4989 (2003).

5. Dettmer, K., Aronov, P.A. \& Hammock, B.D. Mass Spectrom. Rev. 26, 51-78 (2006).

6. Markley, J.L. et al. Pac. Symp. Biocomput. 12 157-168 (2007).

7. Wishart, D.S. et al. Nucleic Acids Res. 35, D521D526 (2007).

8. Weininger, D., Weininger, A. \& Weininger, J.L. J. Chem. Inf. Comput. Sci. 29, 97-101 (1989).

9. Lewis, I.A. et al. Anal. Chem. 79, 9385-9390 (2007).

10. Ulrich, E.L. et al. Nucl. Acids Res. 36, D402-D408 (2008).

\title{
Human Proteinpedia enables sharing of human protein data
}

\section{To the editor:}

Proteomic technologies, such as yeast twohybrid, mass spectrometry (MS), protein/ peptide arrays and fluorescence microscopy, yield multi-dimensional data sets, which are often quite large and either not published or published as supplementary information that is not easily searchable. Without a system in place for standardizing and sharing data, it is not fruitful for the biomedical community to contribute these types of data to centralized repositories. Even more difficult is the annotation and display of pertinent information in the context of the corresponding proteins. Wikipedia, an online encyclopedia that anyone can edit, has already proven quite successful ${ }^{1}$ and can be used as a model for sharing biological data. However, the need for experimental evidence, data standardization and ownership of data creates scientific obstacles.
Here, we describe Human Proteinpedia (http://www.humanproteinpedia.org/) as a portal that overcomes many of these obstacles to provide an integrated view of the human proteome. Human Proteinpedia also allows users to contribute and edit proteomic data with two significant differences from Wikipedia: first, the contributor is expected to provide experimental evidence for the data annotated; and second, only the original contributor can edit their data.

Human Proteinpedia's annotation system provides investigators with multiple options for contributing data including web forms and annotation servers (Supplementary Fig. 1 online). Although registration is required to contribute data, anyone can freely access the data in the repository. The web forms simplify submission through the use of pull-down menus for certain data fields and pop-up menus for standardized vocabulary terms. Distributed annotation servers ${ }^{2}$ using modified protein DAS (distributed annotation system) protocols developed by us (DAS protocols were originally developed for sharing mRNA and DNA data) permit contributing laboratories to maintain protein annotations locally. All protein annotations are visualized in the context of corresponding proteins in the Human Protein Reference Database (HPRD) ${ }^{3}$. Figure 1 shows tissue expression data for alpha-2-HS glycoprotein derived from three different types of experiments.

Our unique effort differs significantly from existing repositories, such as PeptideAtlas ${ }^{4}$ and PRIDE ${ }^{5}$ in several respects. First, most proteomic repositories are restricted to one or two experimental platforms, whereas Human Proteinpedia can accommodate data from diverse platforms, including yeast two-hybrid screens, MS, peptide/protein arrays, immunohistochemistry, western blots, co-immunoprecipitation and fluorescence microscopy-type experiments.

Second, Human Proteinpedia allows contributing laboratories to annotate data pertaining to six features of proteins (posttranslational modifications, tissue expression, cell line expression, subcellular localization, enzyme substrates and protein-protein interactions; Supplementary Fig. 2 online). No existing repository currently permits annotation of all these features in proteins.

Third, all data submitted to Human Proteinpedia are viewable through HPRD in the context of other features of the corresponding proteins. To aid comparison and interpretation, meta-annotations pertaining to samples, method of isolation and experimental platform-specific information are provided (e.g., labeling method, protease used, ionization method, details of primary antibody used).

And fourth, in spite of accommodating multiple data types, the data submission is simplified. This means that a biologist with no technical expertise can login and contribute data.

Thus far, a considerable body of data has been contributed to Human Proteinpedia by the community (see Table 1 ), with a total of $>1.8$ million peptides and $>4$ million MS/MS-spectra deposited. The abovementioned data were derived from 2,695 individual experiments (single experiments are defined as immunohistochemistry performed with a specific antibody, a single MS run or a yeast two-hybrid screen). We have imported MS data from two Human Proteome Organization initiatives, including the human plasma proteome project (HPPP) ${ }^{6}$ and the human liver 


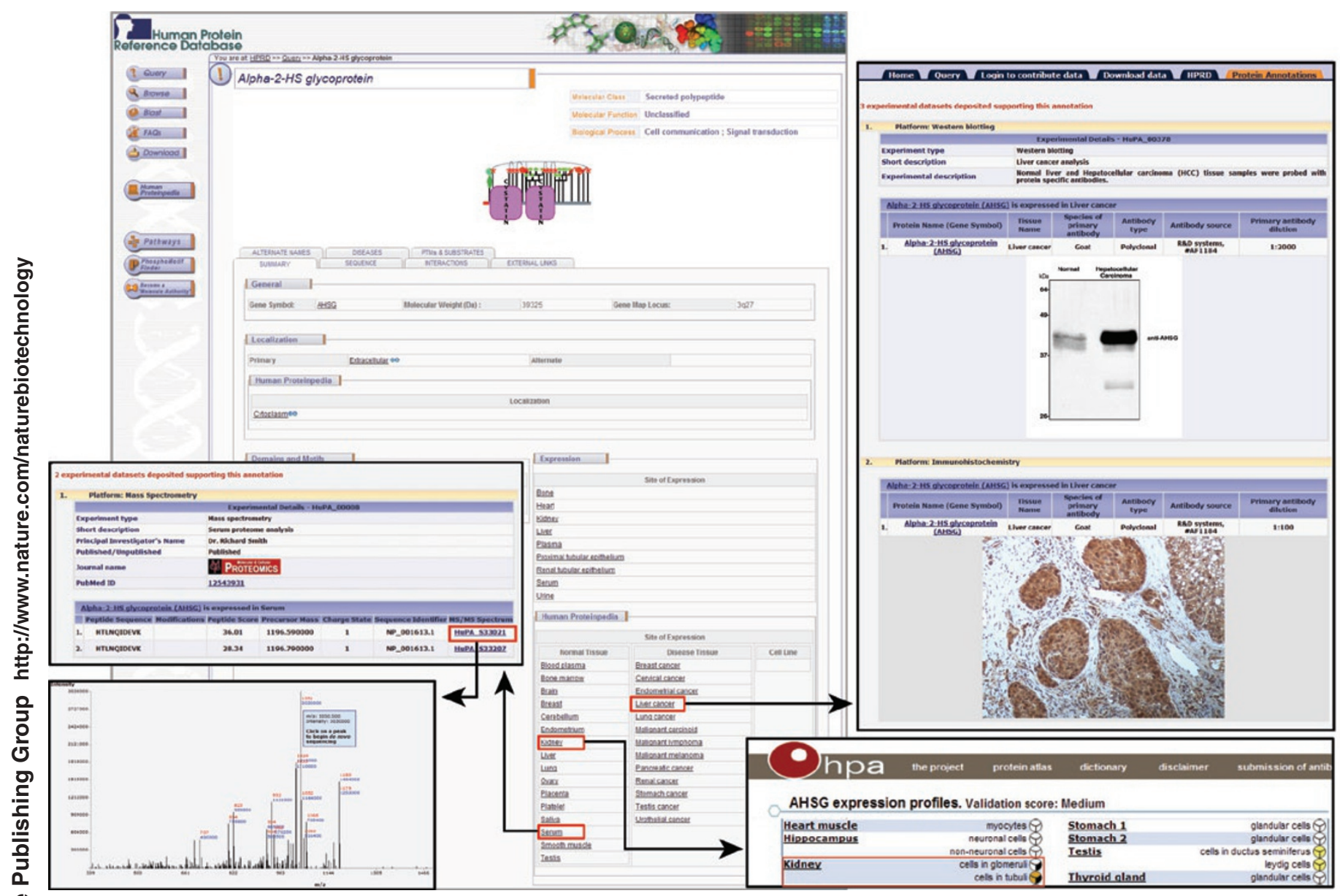

Figure 1 Display of tissue expression data obtained from immunohistochemistry, mass spectrometry and western blot analysis that were submitted to Human Proteinpedia. The molecule page for $\alpha-2-H S$ glycoprotein in Human Protein Reference Database is shown. Annotations pertaining to sites of tissue expression from three types of experimental platforms contributed to Human Proteinpedia are shown. The meta-annotation of a mass spectrometry experiment confirming expression in serum is shown (lower left) along with the corresponding MS/MS spectrum for a peptide displayed using a spectrum viewer obtained from PRIDE. Expression of $\alpha-2$-HS glycoprotein in liver cancer is provided by two entries: one from western blotting (top right) and the other from (2) immunohistochemical labeling, each detailing the antibody used. Expression in kidney is hyperlinked to the corresponding page in the Human Protein Atlas, which contains data from immunohistochemical labeling experiments (lower right).

proteome project (HLPP) ${ }^{7}$. Data from other initiatives like the Human Protein Atlas ${ }^{8}$, Human Unidentified Gene-Encoded (HUGE) project ${ }^{9}$ and LIFEdb ${ }^{10}$ are also included in this effort. The vocabulary follows accepted community standards, such as eVOC ${ }^{11}$, Gene Ontology ${ }^{12}$, RESID $^{13}$, PSI-MI ${ }^{14}$ and PSI-MS.

All data in Human Proteinpedia are freely available to the community for downloading. Storage and dissemination of the raw and processed MS data sets is through the

\begin{tabular}{lc} 
Table 1 Statistics of submitted data & Number \\
Data type & 71 \\
\hline Individual laboratories submitting data & 2,695 \\
Experiments submitted & 203,293 \\
Protein annotations & $4,567,235$ \\
MS/MS spectra & $1,851,124$ \\
Peptide sequences deposited & 138,487 \\
Protein expression & 17,108 \\
Post-translational modifications & 31,476 \\
Protein-protein interactions & 2,906 \\
Subcellular localizations & 16,062 \\
\hline Phosphorylation &
\end{tabular}

Tranche file-sharing network supporting ProteomeCommons.org ${ }^{15}$ (http://www. proteomecommons.org/dev/dfs/) whereas the remainder of the data is available directly from Proteinpedia website. The MS data sets are currently hosted by over 16 servers ( $~ 50$ TB of aggregate capacity) in triplicate including three servers (in India, Japan and the United States) set up especially for this initiative (Supplementary Fig. 1).

Four years of continued manual curation of the scientific literature has yielded $>228,800$ protein annotations in HPRD. The information content in Proteinpedia collected over the course of the past year alone has resulted in the addition of roughly an equal number of entries. Although large collaborations for genome projects are now commonplace, this collaborative initiative of 71 participating laboratories is unprecedented for proteomic data. We are now beginning to contact authors of 
published proteomic studies in a systematic fashion requesting their participation. We eventually hope to capture most of the published data sets, which could be facilitated if depositing experimental data to a public repository is made mandatory for publication, as is already the case for nucleotide sequences, gene expression profiles and protein structures. In this regard, we have instituted a mechanism that allows referees to access data sets submitted by authors before publication in an authorized and anonymous fashion for evaluation purposes.
Note: Supplementary information is available on the Nature Biotechnology website.

\section{ACKNOWLEDGMENTS}

This project has been funded in part by a grant from the National Institutes of Health Roadmap initiative U54 RR020839 (J.S.B., P.A.C., R.J.C. and A.P.) and a contract N01-HV-28180 from the National Heart Lung and Blood Institute (J.V.E., R.J.C. and A.P.). The content of this publication does not necessarily reflect the views or policies of the Department of Health and Human Services, nor does mention of trade names, commercial products, or organization imply endorsement by the United States Government.

1 Giles, J. Nature 438, 900-901 (2005)

2. Dowell, R.D., Jokerst, R.M., Day, A., Eddy, S.R. \& Stein, L. BMC Bioinformatics 2, 7 (2001)
3. Peri, S. et al. Genome Res. 13, 2363-2371 (2003).

4. Desiere, F. et al. Genome Biol.6, R9 (2005).

5. Martens, L. et al. Proteomics 5, 3537-3545 (2005).

6. States, D.J. et al. Nat. Biotechnol. 24, 333-338 (2006).

7. He, F. Mol Cell Proteomics 4, 1841-1848 (2005).

8. Uhlen, M. et al. Mol. Cell Proteomics 4, 1920-1932 (2005).

9. Nakajima, D. et al. DNA Res 12, 257-267 (2005).

10. Mehrle, A. et al. Nucleic Acids Res. 34, D415-D418 (2006).

11. Kelso, J. et al. Genome Res. 13, 1222-1230 (2003).

12. Ashburner, M. et al. Nat. Genet. 25, 25-29 (2000).

13. Garavelli, J.S. Nucleic Acids Res. 31, 499-501 (2003).

14. Hermjakob, H. et al. Nat. Biotechnol. 22, 177-183 (2004).

15. Falkner, J.A., Falkner, J.W. \& Andrews, P.C. Bioinformatics 23, 262-263 (2007)

Suresh Mathivanan ${ }^{1-4}$, Mukhtar Ahmed ${ }^{1}$, Natalie G Ahn ${ }^{5}$, Hainard Alexandre ${ }^{6}$, Ramars Amanchy ${ }^{2,3}$, Philip C Andrews ${ }^{7}$, Joel S Bader ${ }^{8,9}$,

Brian M Balgley ${ }^{10}$, Marcus Bantscheff ${ }^{11}$, Keiryn L Bennett ${ }^{12}$, Erik Björling ${ }^{13}$, Blagoy Blagoev ${ }^{14}$, Ron Bose ${ }^{15}$, Samir K Brahmachari ${ }^{16}$, Alma S Burlingame ${ }^{17}$, Xosé R Bustelo $^{18}$, Gerard Cagney ${ }^{19}$, Greg T Cantin ${ }^{20}$, Helene L Cardasis ${ }^{21}$, Julio E Celis ${ }^{22}$, Raghothama Chaerkady ${ }^{1,2}$, Feixia Chu ${ }^{17}$, Philip A Cole ${ }^{15}$, Catherine E Costello ${ }^{23}$, Robert J Cotter ${ }^{24}$, David Crockett ${ }^{25}$, James P DeLany ${ }^{26}$, Angelo M De Marzo ${ }^{27}$, Leroi V DeSouza ${ }^{28}$, Eric W Deutsch ${ }^{29}$, Eric Dransfield ${ }^{30}$, Gerard Drewes ${ }^{11}$, Arnaud Droit ${ }^{31}$, Michael J Dunn ${ }^{19}$, Kojo Elenitoba-Johnson ${ }^{25}$, Rob M Ewing ${ }^{32,33}$, Jennifer Van Eyk ${ }^{34}$, Vitor Faca ${ }^{35}$, Jayson Falkner ${ }^{7}$, Xiangming Fang ${ }^{36}$, Catherine Fenselau ${ }^{37}$, Daniel Figeys ${ }^{38}$, Pierre Gagne ${ }^{31}$, Cecilia Gelfi ${ }^{39}$, Kris Gevaert ${ }^{40}$, Jeffrey M Gimble ${ }^{41,42}$, Florian Gnad ${ }^{43}$, Renu Goel ${ }^{1}$, Pavel Gromov ${ }^{22}$, Samir M Hanash ${ }^{35}$, William S Hancock ${ }^{44}$, HC Harsha ${ }^{1-3}$, Gerald Hart ${ }^{3}$, Faith Hays ${ }^{37}$, Fuchu He ${ }^{45,46}$, Prashantha Hebbar ${ }^{1}$, Kenny Helsens ${ }^{40}$, Heiko Hermeking ${ }^{47}$, Winston Hide ${ }^{48}$, Karin Hjern $\varnothing^{14}$, Denis F Hochstrasser ${ }^{6,49}$, Oliver Hofmann $^{48}$,

David M Horn ${ }^{50}$, Ralph H Hruban ${ }^{51}$, Nieves Ibarrola ${ }^{18}$, Peter James ${ }^{52}$, Ole N Jensen ${ }^{14}$, Pia Hønnerup Jensen ${ }^{14}$, Peter Jung ${ }^{47}$, Kumaran Kandasamy ${ }^{1-4}$, Indu Kheterpal ${ }^{53}$, Reiko F Kikuno ${ }^{54}$, Ulrike Korf ${ }^{55}$, Roman Körner ${ }^{56}$, Bernhard Kuster ${ }^{11}$, Min-Seok Kwon ${ }^{57}$, Hyoung-Joo Lee ${ }^{57}$, Young-Jin Lee ${ }^{58}$, Michael Lefevre ${ }^{41,59}$, Minna Lehvaslaiho ${ }^{48}$, Pierre Lescuyer ${ }^{6}$, Fredrik Levander ${ }^{52}$, Megan S Lim $^{25}$, Christian Löbke ${ }^{55}$, Joseph A Loo ${ }^{60}$, Matthias Mann $^{14,43}$, Lennart Martens ${ }^{40,61}$, Juan Martinez-Heredia ${ }^{62}$, Mark McComb ${ }^{23}$, James McRedmond ${ }^{19}$, Alexander Mehrle ${ }^{55}$, Rajasree Menon ${ }^{63}$, Christine A Miller ${ }^{50}$,

of Harald Mischak ${ }^{64}$, S Sujatha Mohan ${ }^{1}$, Riaz Mohmood ${ }^{4}$, Henrik Molina ${ }^{2,3}$, Michael F Moran ${ }^{65,66}$, James D Morgan ${ }^{27}$, Robert Moritz ${ }^{67}$, Martine Morzel ${ }^{68}$, David C Muddiman ${ }^{69}$, Anuradha Nalli ${ }^{1-3}$, J Daniel Navarro ${ }^{1}$, Thomas A Neubert ${ }^{21}$, Osamu Ohara ${ }^{54,70}$, Rafael Oliva ${ }^{62}$, Gilbert S Omenn ${ }^{71}$, Masaaki Oyama ${ }^{72}$, Young-Ki Paik ${ }^{57}$, Kyla Pennington ${ }^{19}$, Rainer Pepperkok ${ }^{73}$, Balamurugan Periaswamy ${ }^{1-4}$, Emanuel F Petricoin ${ }^{74}$, Guy G Poirier $^{31}$, TS Keshava Prasad ${ }^{1}$, Samuel O Purvine ${ }^{75}$, B Abdul Rahiman ${ }^{4}$, Prasanna Ramachandran ${ }^{60}$, Y L Ramachandra ${ }^{4}$, Robert H Rice ${ }^{58}$, Jens Rick ${ }^{11}$, Ragna H Ronnholm ${ }^{76}$, Johanna Salonen ${ }^{77}$, Jean-Charles Sanchez ${ }^{6}$, Thierry Sayd ${ }^{30}$, Beerelli Seshi ${ }^{78}$, Kripa Shankari ${ }^{1}$, Shi Jun Sheng ${ }^{34}$, Vivekananda Shetty $^{21}, K_{\text {S Shivakumar }}{ }^{1,4}$, Richard J Simpson ${ }^{67}$, Ravi Sirdeshmukh ${ }^{79}, \mathrm{~K}$ W Michael Siu ${ }^{28}$, Jeffrey C Smith ${ }^{38}$, Richard D Smith ${ }^{75}$, David J States $^{63}$, Sumio Sugano ${ }^{72}$, Matthew Sullivan ${ }^{19}$, Giulio Superti-Furga ${ }^{12}$, Maarit Takatalo ${ }^{76}$, Visith Thongboonkerd ${ }^{80}$, Jonathan C Trinidad ${ }^{17}$, Mathias Uhlen ${ }^{13}$, Joël Vandekerckhove ${ }^{40}$, Julian Vasilescu ${ }^{38}$, Timothy D Veenstra ${ }^{81}$, José-Manuel Vidal-Taboada ${ }^{62}$, Mauno Vihinen ${ }^{77}$, Robin Wait ${ }^{82}$, Xiaoyue Wang ${ }^{3}$, Stefan Wiemann ${ }^{55}$, Billy Wu ${ }^{44}$, Tao Xu ${ }^{20}$, John R Yates ${ }^{20}$, Jun Zhong ${ }^{2,3}$, Ming Zhou ${ }^{81}$, Yunping Zhu ${ }^{45}$, Petra Zurbig ${ }^{64}$ and Akhilesh Pandey ${ }^{2,3}$

The authors are listed alphabetically by their last name except for the first (S.M.) and the last (A.P.) authors.

${ }^{1}$ Institute of Bioinformatics, International Tech Park, Bangalore 560 066, India. ${ }^{2}$ McKusick-Nathans Institute of Genetic Medicine and the Departments of Pathology and Oncology, Johns Hopkins University, Baltimore, Maryland 21205, USA. ${ }^{3}$ Department of Biological Chemistry, Johns Hopkins University School of Medicine, Baltimore, Maryland 21205, USA. ${ }^{4}$ Department of Biotechnology, Kuvempu University, Shankaraghatta 577451, Karnataka, India. ${ }^{5}$ Department of Chemistry and Biochemistry, Howard Hughes Medical Institute, University of Colorado, Boulder, Colorado 80309, USA. ${ }^{6}$ Biomedical Proteomics Research Group, Department of Structural Biology and Bioinformatics, Geneva University, Faculty of Medicine, CH-1211 Geneva 4, Switzerland. ${ }^{7}$ Department of Biological Chemistry, University of Colorado, Ann Arbor, Colorado 48109-0674, USA. ${ }^{8}$ The High Throughput Biology Center, Johns Hopkins University, Baltimore, Maryland 21205, USA. ${ }^{9}$ Department of Biomedical Engineering, Johns Hopkins University, Baltimore, Maryland 21205, USA. ${ }^{10}$ Calibrant Biosystems, 910 Clopper Road, Suite 220N, Gaithersburg, Maryland 20878, USA. ${ }^{11}$ Cellzome AG, Meyerhofstrasse 1, 69117 Heidelberg, Germany. ${ }^{12}$ Research Center for Molecular Medicine (CeMM), Lazarettgasse 19/3, 1090 Vienna, Austria. ${ }^{13}$ Royal Institute of Technology, School of Biotechnology, AlbaNova University Center, SE-106, 91 Stockholm, Sweden. ${ }^{14}$ Department of Biochemistry and Molecular Biology, University of Southern Denmark, Campusvej 55, DK-5230 Odense M, Denmark. ${ }^{15}$ Departments of Pharmacology and Oncology, Johns Hopkins University School of Medicine, Baltimore, Maryland 21205, USA. ${ }^{16}$ GN Ramachandran Knowledge Center for Genome Informatics, Institute of Genomics and Integrative Biology, CSIR, Mall Road, Delhi 110 007, India. ${ }^{17}$ Department of Pharmaceutical Chemistry, University of California-San Francisco, San Francisco, California $94143-$ 0446, USA. ${ }^{18}$ Centro de Investigación del Cáncer and Instituto de Biología Molecular y Celular del Cáncer. CSIC-University of Salamanca, Campus Unamuno, E-37007 Salamanca, Spain. ${ }^{19}$ Proteome Research Centre, UCD Conway Institute of Biomolecular and Biomedical Research, Belfield, Dublin 4, Ireland. ${ }^{20}$ Department of Cell Biology, The Scripps Research Institute, 10550 North Torrey Pines Road, La Jolla, California 92037, USA. ${ }^{21}$ Skirball Institute of Biomolecular Medicine and Department of Pharmacology, New York University School of Medicine, New York, New York 10016, USA. ${ }^{22}$ Danish Centre for Translational Breast Cancer Research (DCTB) and Department of Proteomics in Cancer, Institute of Cancer Biology, Danish Cancer Society, Strandboulevarden 49, DK-2100, Copenhagen, Denmark. ${ }^{23}$ Cardiovascular Proteomics Center, Boston University School of Medicine, Boston, Massachusetts 02118, USA. ${ }^{24}$ Department of Pharmacology and Molecular Sciences, Johns Hopkins University School of Medicine, Baltimore, Maryland 21205, USA. ${ }^{25}$ Department of Pathology, University of Michigan Medical School in Ann Arbor, 1301 Catherine 5240 Medical Science I, Ann Arbor, Michigan 48109-0602, USA. ${ }^{26}$ Endocrinology and Metabolism Division, Department of Medicine, University of Pittsburgh, Pittsburgh, Pennsylvania 15213, USA. ${ }^{27}$ Departments of Urology and Pathology, The Johns Hopkins University School of Medicine, 600 North Wolfe Street, Baltimore, Maryland 21287, USA. ${ }^{28}$ Centre for Research in Mass Spectrometry and Department of Chemistry, York University, 4700 Keele Street, Toronto, Ontario M3J 1P3, 
Canada. ${ }^{29}$ Institute for Systems Biology, Seattle, Washington 98103, USA. ${ }^{30}$ Wageningen Centre for Food Sciences, Diedenweg 20, PO Box 557, 6700 AN Wageningen, The Netherlands. ${ }^{31}$ Health and Environment Unit, Laval University Medical Research Center (CHUL), Faculty of Medicine, 2705 Boul. Laurier, Quebec City, QC, G1V 4G2, Canada. ${ }^{32}$ Protana (now Transition Therapeutics), 101 College Street, Suite 220, Toronto, Ontario, M5G 1L7, Canada. ${ }^{33}$ Infochromics, MaRS Discovery District, 101 College St., Toronto, Ontario M5G 1L7, Canada. ${ }^{34}$ Department of Medicine, Johns Hopkins Bayview Proteomics Center, Johns Hopkins University, Baltimore, Maryland 21224. ${ }^{35}$ Fred Hutchinson Cancer Research Center, Seattle, Washington 98109 , USA. ${ }^{36}$ GenWay Biotech, Inc., 6777 Nancy Ridge Drive, San Diego, California 92121, USA. ${ }^{37}$ Department of Chemistry and Biochemistry, University of Maryland, College Park, Maryland 20742, USA. ${ }^{38}$ The Ottawa Institute of Systems Biology, University of Ottawa, 451 Smyth Road, Ottawa, Ontario, K1H 8 M5 Canada. ${ }^{39}$ Department of Biomedical Sciences and Technologies, Faculty of Medicine, University of Milano, Via Fratelli Cervi 93, Segrate Milano, Italy. ${ }^{40}$ Department of Biochemistry and Medical Protein Research, Faculty of Medicine and Health Sciences, Ghent University, A. Baertsoenkaai 3, B9000

하 Ghent, Belgium. ${ }^{41}$ Stem Cell Laboratory, Pennington Biomedical Research Center, Louisiana State University System, Baton Rouge, Louisiana 70808, USA.

을 ${ }^{42}$ Cell Biology Core Facility, Pennington Biomedical Research Center, Louisiana State University System, Baton Rouge, Louisiana 70808, USA.

${ }^{43}$ Department of Proteomics and Signal Transduction, Max-Planck-Institute for Biochemistry, D-82152 Martinsried, Germany. ${ }^{44}$ Barnett Institute and Department of Chemistry and Chemical Biology, Northeastern University, Boston, Massachusetts 02115, USA. ${ }^{45}$ Beijing Proteome Research Center, Beijing Institute of Radiation Medicine, Beijing, 100850, China. ${ }^{46}$ Institutes of Biomedical Sciences, Fudan University, Shanghai, 200032, China. ${ }^{47}$ Molecular Oncology, Independent Max-Planck-Research Group, Max-Planck-Institute of Biochemistry, Am Klopferspitz 18, D-82152 Martinsried, Germany. ${ }^{48}$ South African National Bioinformatics Institute, University of the Western Cape Bellville 7535, South Africa. ${ }^{49}$ Central Clinical Chemistry Laboratory, Geneva University Hospital, Geneva, Switzerland. ${ }^{50}$ Agilent Technologies, 5301 Stevens Creek Blvd., Santa Clara, California 95051, USA. ${ }^{51}$ Department of Pathology and Oncology, The Sol Goldman Pancreatic Cancer Research Center, Johns Hopkins Medical Institutions, 401 North Broadway, Weinberg 2242, Baltimore, Maryland 21231-2410, USA. ${ }^{52}$ Protein Technology, Lund University, BMC D13, Lund, Sweden. ${ }^{53}$ Proteomic Core Facility, Pennington Biomedical Research Center, Louisiana State University System, Baton Rouge, Louisiana 70808, USA. ${ }^{54}$ Department of Human Genome Technology, Kazusa DNA Research Institute, 2-6-7 Kazusa-kamatari Kisarazu Chiba 292-081,8 Japan. ${ }^{55}$ Division of Molecular Genome Analysis, German Cancer Research Center-DKFZ Heidelberg, Im Neuenheimer Feld 580, 69120 Heidelberg, Germany. ${ }^{56}$ Department of Cell Biology, Max Planck Institute of Biochemistry, D-82152 Martinsried, Germany. ${ }^{57}$ Department of Biochemistry, Yonsei Proteome Research Center and Biomedical Proteome Research Center, Yonsei University, Seoul 120-749, South Korea. ${ }^{58}$ Department of Environmental Toxicology, University of California, Davis, California 95616-8588, USA.

${ }^{59}$ Lipoprotein Laboratory, Pennington Biomedical Research Center, Louisiana State University System, Baton Rouge, Louisiana 70808, USA. ${ }^{60}$ Department of Chemistry and Biochemistry and Department of Biological Chemistry, University of California Los Angeles, Los Angeles, California 90095, USA. ${ }^{61}$ EMBL 으 Outstation, The European Bioinformatics Institute, Wellcome Trust Genome Campus, Cambridge CB10 1SD, UK. ${ }^{62}$ Human Genetics Research Group, IDIBAPS, Faculty of Medicine, University of Barcelona, Casanova 143, 08023, Barcelona, Spain. ${ }^{63}$ Bioinformatics Program, University of Michigan, Ann Arbor, Michigan 48109, USA. ${ }^{64}$ Mosaiques Diagnostics \& Therapeutics AG, Hanover, Mellendorfer Strasse 7-9D-30625 Hannover, Germany. ${ }^{65}$ Cancer O Program, Hospital For Sick Children and McLaughlin Centre for Molecular Medicine, 101 College Street, East Tower, Toronto, Ontario, M5G 1L7 Canada. ${ }^{66}$ Banting and Best Department of Medical Research and Department of Molecular and Medical Genetics, University of Toronto, 112 College St., Toronto, Ontario M5G 1L6, Canada. ${ }^{67}$ Joint Proteomics Laboratory, Ludwig Institute for Cancer Research (Melbourne Branch) and The Walter and Eliza Hall Institute of Medical Research, 1 G Royal Parade, Parkville, 3050 Victoria, Australia. ${ }^{68}$ INRA, UR 370, Proteomics Platform, 63122 Saint Genès Champanelle, France. ${ }^{69}$ W.M. Keck FT-ICR Mass Spectrometry Laboratory, Department of Chemistry, North Carolina State University, Raleigh, North Carolina 27695, USA. ${ }^{70}$ Laboratory for Immunogenomics, RIKEN Research Center for Allergy and Immunology, RIKEN Yokohama Institute, 2nd floor, North Research Building, 1-7-22 Suehiro-cho, Tsurumi-ku, Yokohama City, Kanagawa, 230-0045, Japan. ${ }^{71}$ Department of Internal Medicine, Center for Computational Medicine and Biology and Proteomics Alliance for Cancer Research, University of Michigan, Ann Arbor, Michigan 48109-2218, USA. ${ }^{72}$ Medical Proteomics Laboratory, Institute of Medical Science, University of Tokyo, Minato-ku, Tokyo 108-8639, Japan. ${ }^{73}$ European Molecular Biology Laboratory, Cell Biology and Biophysics Programme Meyerhofstrasse 1, D-69117 Heidelberg, Germany. ${ }^{74}$ Center for Applied Proteomics and Molecular Medicine, George Mason N University, Manassas, Virginia 20110, USA. ${ }^{75}$ Environmental Molecular Science Laboratory and Biological Sciences Division, Pacific Northwest National Laboratory, Richland, Washington 99352, USA. ${ }^{76}$ Department of the Biological and Environmental Sciences, Division of Biochemistry, University of

Do Helsinki, PO Box 56, Viikinkaari 9, FI-00014, Finland. ${ }^{77}$ Institute of Medical Technology, University of Tampere, Finn-Medi2, $5^{\text {th }}$ floor, Biokatu 8, Tampere, FI-33014, Finland. ${ }^{78}$ Department of Pathology, Los Angeles Biomedical Research Institute at Harbor-UCLA Medical Center, David Geffen School of Medicine at UCLA, Torrance, California 90502-2064, USA. ${ }^{79}$ Centre for Cellular and Molecular Biology, Uppal Road, Hyderabad 500 007, India. ${ }^{80}$ Medical Molecular Biology Unit, Office for Research and Development, Faculty of Medicine Siriraj Hospital, Mahidol University, Bangkok 10700, Thailand. ${ }^{81}$ Laboratory of Proteomics and Analytical Technologies, SAIC-Frederick Inc., NCI-Frederick, Frederick, Maryland 21702, USA. ${ }^{82}$ Kennedy Institute of Rheumatology, Faculty of Medicine, Imperial College, South Kensington Campus, London SW7 2AZ, UK. e-mail:pandey@jhmi.edu 